\title{
CCD photometry and distances of six resolved irregular galaxies in Canes Venatici
}

\author{
N.A. Tikhonov and I.D. Karachentsev \\ Special Astrophysical Observatory, Russian Academy of Sciences, N. Arkhyz, Stavropolsky kraj, 357147, Russia
}

Received June 23; accepted June 25, 1997

\begin{abstract}
The results of detailed $B, V$ imaging of six nearby irregular galaxies with radial velocities $V_{0}<$ $300 \mathrm{~km} / \mathrm{s}$ are presented. Except for one all the galaxies have been resolved into stars for the first time. Based on photometry of the brightest blue stars we derived the following distances to the galaxies: 3.6 Mpc for NGC 4163, 3.5 Mpc for NGC 4190, 8.6 Mpc for UGC 7298, 4.8 Mpc for UGC 7577, $3.7 \mathrm{Mpc}$ for UGC 8308, and 4.0 Mpc for UGC 8320.
\end{abstract}

Key words: galaxies: irregular — galaxies: distances galaxies: stellar content

\section{Introduction}

This article continues a series of publications (Georgiev et al. 1997; Makarova et al. 1997; Makarova et al. 1998) devoted to the study of the 3D- structure of the nearest scattered complex of galaxies in Canes Venatici $(=\mathrm{CVn})$. Being situated near the Local Supercluster equator, the CVn cloud is projected onto other galaxy groups, which complicates the analysis of its structure and kinematics. About $90 \%$ of the population of the CVn cloud are irregular dwarf galaxies. Application of the Tully \& Fisher (1977) method to them gives distance modulus estimates with a rather low accuracy: $\sigma(m-M)=1.0-1.5$ mag.

In large-scale images of irregular galaxies in several bands one can distinguish blue supergiants and use their luminosity to determine distance moduli with a typical error of about 0.4 mag (Karachentsev \& Tikhonov 1994). All the six irregular galaxies studied here have corrected radial velocities $V_{0}<300 \mathrm{~km} / \mathrm{s}$, suggesting close distances. The results of our photometry confirm this suggestion.

\section{Observations and photometry}

Images of the six observed irregular dwarf galaxies are reproduced from the Digital POSS in Fig. 1. We performed observations of the galaxies in March 10, 1994 at the prime focus of the 6 meter telescope (scale $8.6 \mathrm{arcsec} / \mathrm{mm}$ ). A CCD chip of $580 \times 520$ pixels was used with a readout noise of 12 e and a pixel size of $0.18 \times 0.24$ microns, which yielded a total field of $120 \times 80 \mathrm{arcsec}$. For each of the galaxies we obtained $B$ and $V$ frames with a typical exposure $\sim 600 \mathrm{~s}$ under a seeing of $F W H M=1.3-1.5$ arcsec. The frames were calibrated using the equatorial standard stars from Landolt (1992).

After the initial processing of the frames we made aperture photometry of stars using the PC VISTA package (Treffers \& Richmond 1990) and the UMAG package of Georgiev (1995), which allows us to reduce the inhomogeneity of the local background around the measured stars.

The $V$ images of the observed galaxies are presented in Fig. 2. To show the structure of the central part as well as of the periphery of each galaxy, we subtracted a frame smoothed with a window of $10 \times F W H M$. Based on the results of our photometry the Colour-Magnitude diagrams were derived for the brightest stars of the galaxies. We reproduce them in Fig. 2, where star-like objects are indicated with filled squares and slightly diffuse ones with open squares. The brightest blue $(B-V<0.4)$ and red $(B-V>1.6)$ stars are marked in Fig. 2. Their apparent $B$ magnitudes and $B-V$ colours are presented in Table 1. The complete tables of the photometric data are accessible via FTP address: ntik@sao.ru.

\section{Discussion}

Following the usual approach developed by Sandage \& Tammann (1974) and de Vaucouleurs (1978), we determined the galaxy distance moduli from the mean apparent magnitude of the three brightest blue stars, $\langle B(3 B)\rangle$, from the relation:

$$
\mu_{0}(B)=1.51<B(3 B)>-0.51 B_{\mathrm{t}}-A_{\mathrm{b}}+4.14,
$$

where $B_{\mathrm{t}}$ is the integral magnitude of the parent galaxy, $A_{\mathrm{b}}$ is the galactic extinction, and the numerical coefficients were calibrated (Karachentsev \& Tikhonov 1994) from galaxies whose distances had been measured from 


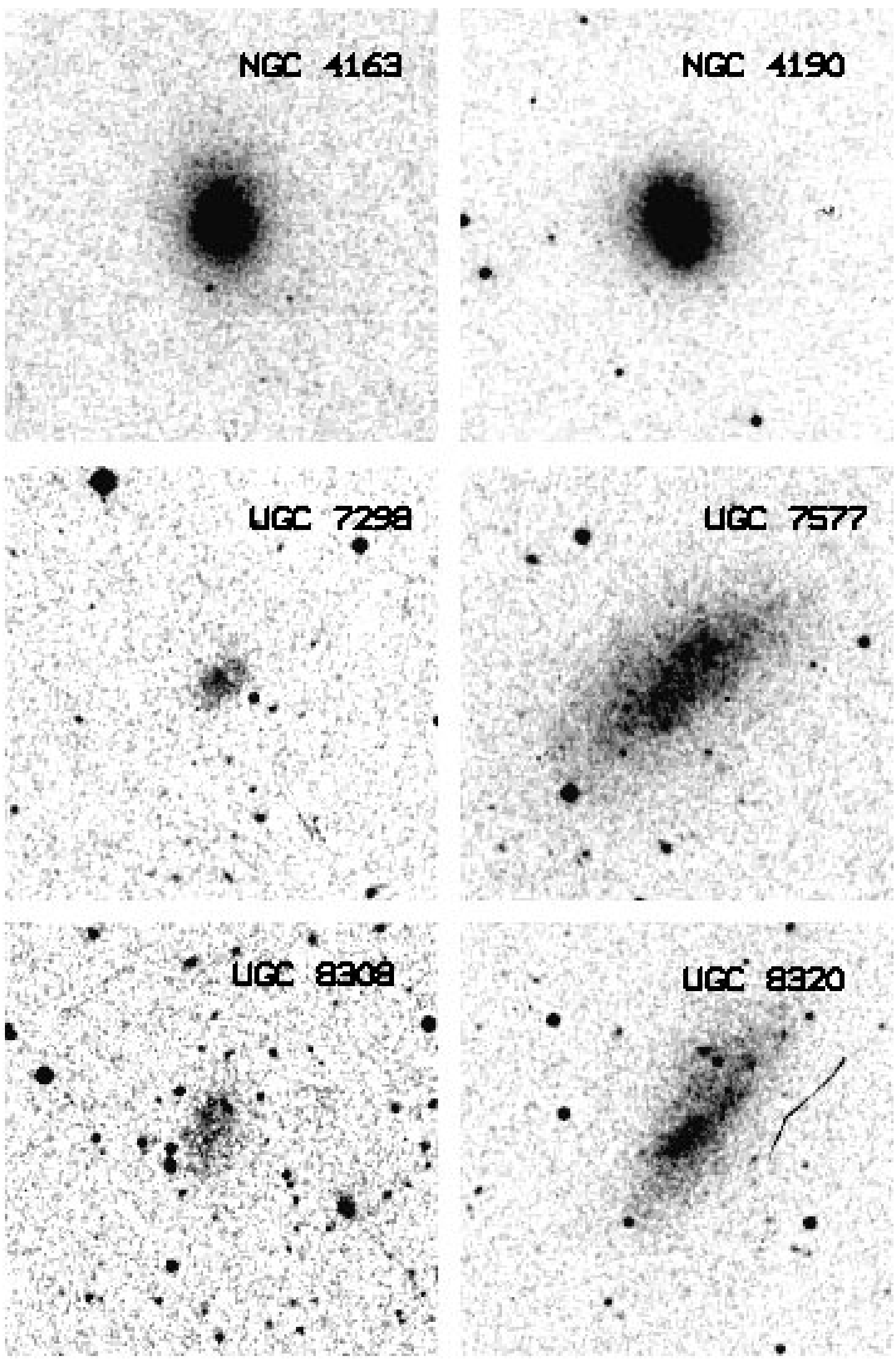

Fig. 1. Reproductions from the Digital POSS-I of the six irregular galaxies. Each chart is $5 \times 5$ arcmin. North at top, East at left 

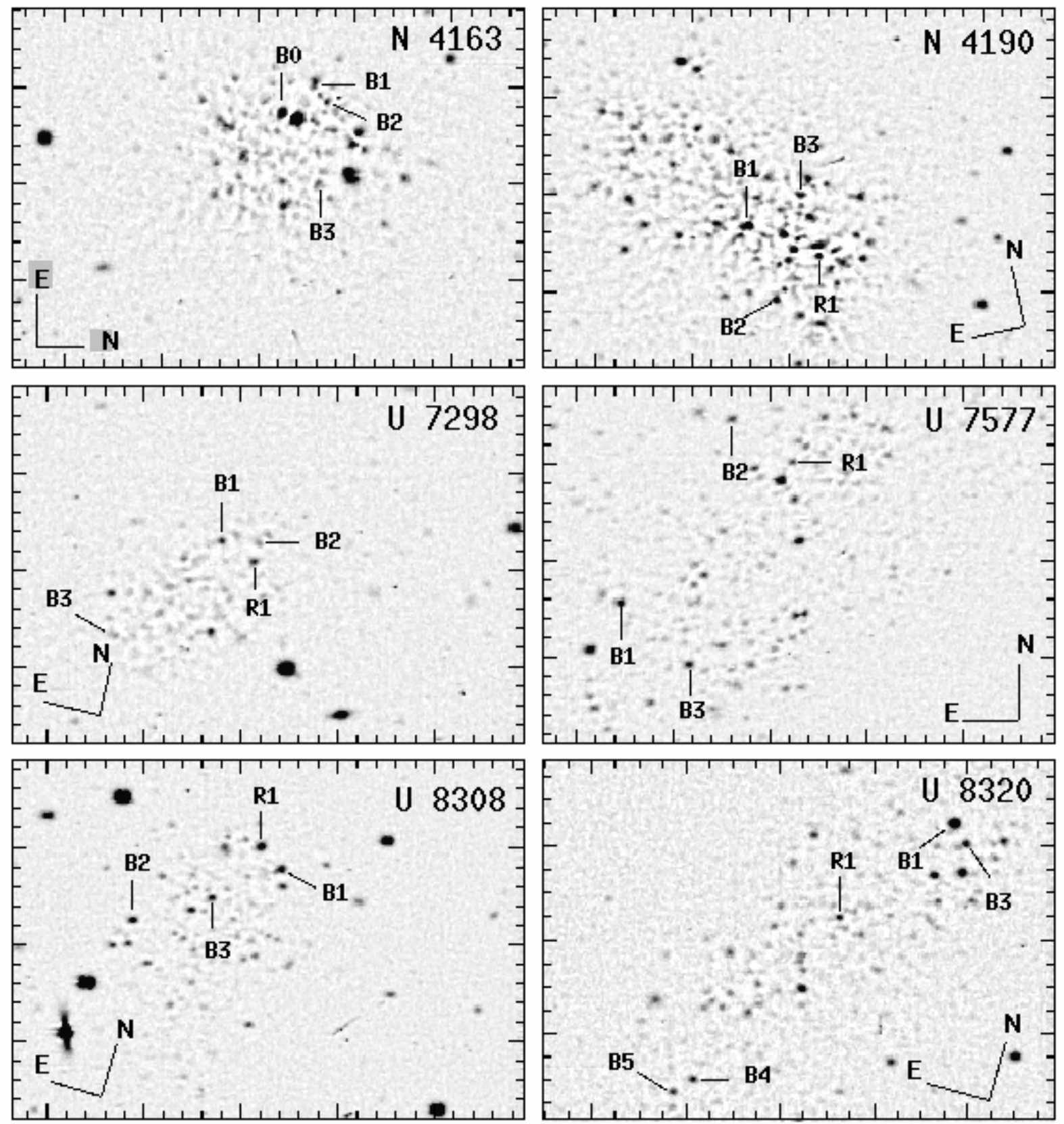

Fig. 2. The $V$ frames of NGC 4163, NGC 4190, UGC 7298, UGC 7577, UGC 8308, and UGC 8320 after a subtraction of a smoothed frame. North and East are indicated by arrows. The brightest stars from Table 1 are marked. Tick marks are given for every 5 arcsec

cepheids. In the cases when we found red supergiant candidates, additional distance estimates were derived from a similar relation:

$\mu_{0}(R)=1.10 V(R 1)-0.10 B_{\mathrm{t}}-0.76 A_{\mathrm{b}}+7.00$

Some basic parameters of the galaxies are presented in Table 2. Its lines contain:

(1) - the standard angular diameter of the galaxy in arcmin from the Catalogue PGC-ROM (Paturel et al. 1992);
(2) - the integrated magnitude of the galaxy either from PGC or from our photometry (marked by "c");

(3) - the integrated colour index of the galaxy within its standard diameter according our measurements;

(4) - the value of the galactic extinction from the Catalogue PGC-ROM;

(5) - the radial velocity (in $\mathrm{km} / \mathrm{s}$ ) corrected for motion with respect to the Local group centroid (Karachentsev \& Makarov 1996); 


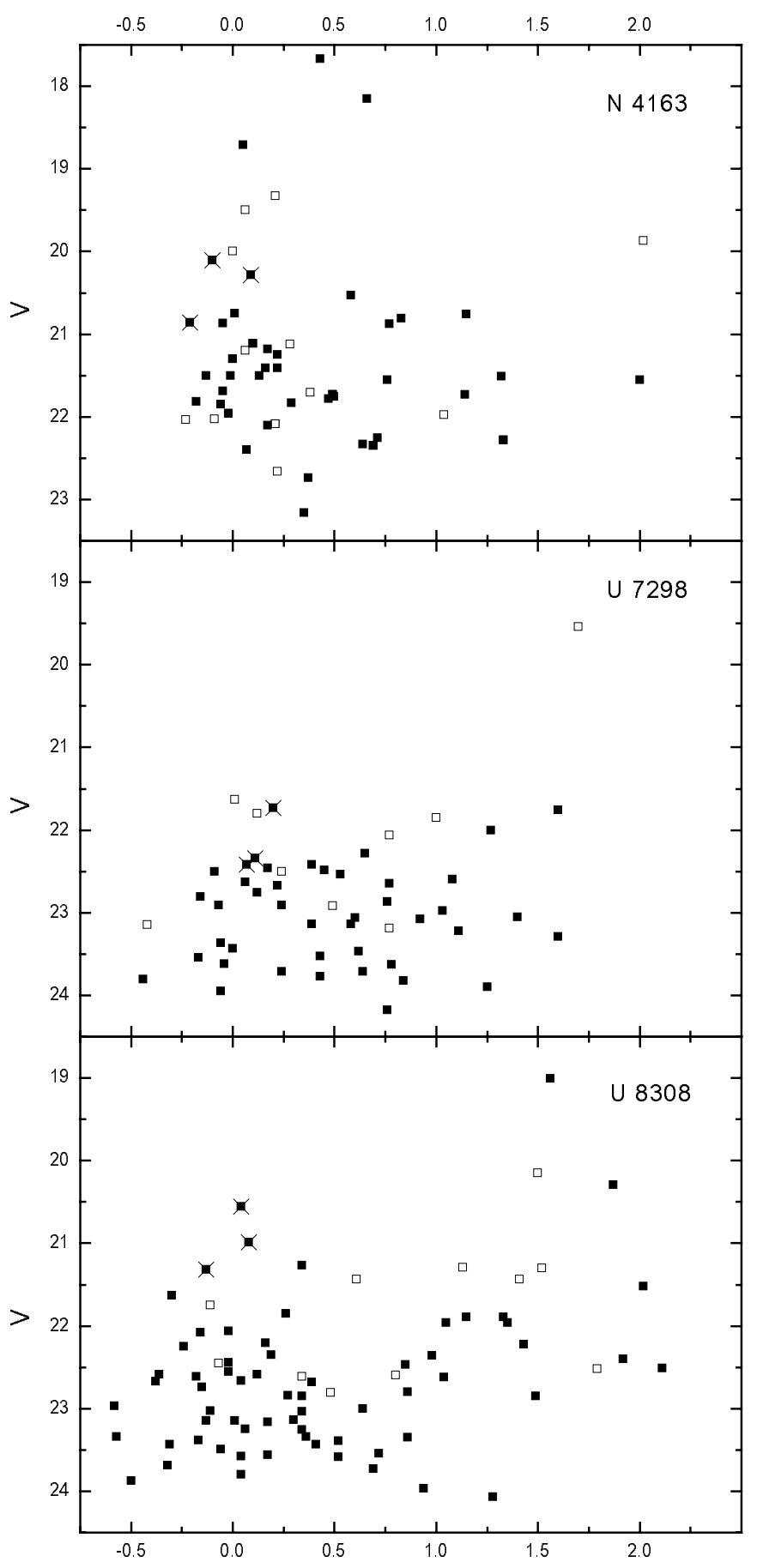

$B-V$

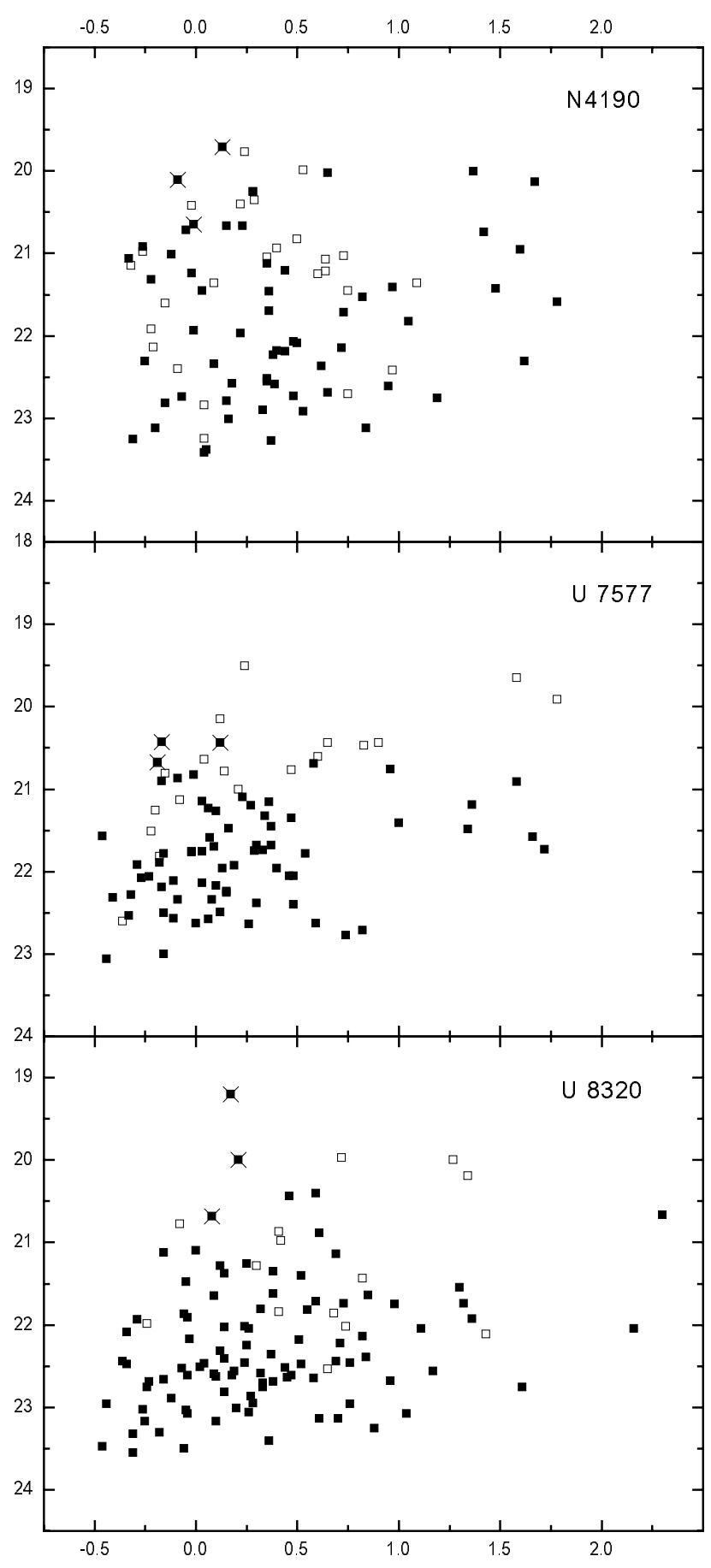

$B-V$

Fig. 3. Colour-Magnitude diagrams for the six dwarf galaxies. Dark squares are star-like objects, open squares are slightly diffuse ones, crosses are the blue stars which have been used for distance determination 
Table 2. Some global properties of the irregular galaxies

\begin{tabular}{|ccccccc|}
\hline Parameter & $\mathrm{N} 4163$ & $\mathrm{~N} 4190$ & $\mathrm{U} 7298$ & $\mathrm{U} 7577$ & $\mathrm{U} 8308$ & $\mathrm{U} 8320$ \\
\hline$a_{25}$ & 1.7 & 1.6 & 1.2 & 4.2 & 1.1 & 3.7 \\
$B_{\mathrm{t}}$ & $13.93 \mathrm{c}$ & $13.52 \mathrm{c}$ & $15.83 \mathrm{c}$ & 12.87 & $15.53 \mathrm{c}$ & 12.73 \\
$(B-V)_{\mathrm{t}}$ & $0.45 \mathrm{c}$ & $0.44 \mathrm{c}$ & $0.40 \mathrm{c}$ & $0.45 \mathrm{c}$ & $0.48 \mathrm{c}$ & $0.31 \mathrm{c}$ \\
$A_{\mathrm{b}}$ & 0.0 & 0.0 & 0.04 & 0.0 & 0.0 & 0.0 \\
$V_{0}$ & 165 & 234 & 253 & 240 & 244 & 273 \\
$<B(3 B)>$ & 20.34 & 20.17 & 22.28 & 20.43 & 20.95 & 20.11 \\
$<B-V>_{3}$ & -0.07 & 0.01 & 0.13 & -0.08 & 0.00 & 0.15 \\
$\mu_{0}(3 B)$ & 27.75 & 27.70 & 29.67 & 28.42 & 27.85 & 28.01 \\
$\mu_{0}(1 R)$ & - & 27.79 & 29.32 & $(28.71)$ & 27.77 & 28.46 \\
$D$ & 3.55 & 3.47 & 8.59 & 4.84 & 3.72 & 4.01 \\
\hline
\end{tabular}

Table 1. $B, V$ photometry of the brightest stars in six irregular galaxies

\begin{tabular}{|c|c|c|c|c|c|}
\hline star & $B$ & $B-V$ & star & $B$ & $B-V$ \\
\hline \multicolumn{3}{|c|}{ NGC 4163} & \multicolumn{3}{|c|}{ UGC 7577} \\
\hline B0 & 18.76 & 0.05 & B1 & 20.26 & -0.17 \\
\hline B1 & 20.01 & -0.10 & B2 & 20.48 & -0.19 \\
\hline B2 & 20.37 & 0.09 & B3 & 20.56 & 0.12 \\
\hline B3 & 20.65 & -0.21 & (R1) & 22.49 & 1.58 \\
\hline \multicolumn{3}{|c|}{ NGC 4190} & \multicolumn{3}{|c|}{ UGC 8308} \\
\hline B1 & 19.84 & 0.13 & B1 & 20.60 & 0.04 \\
\hline B2 & 20.02 & -0.09 & B2 & 21.07 & 0.08 \\
\hline B3 & 20.64 & -0.01 & B3 & 21.19 & -0.13 \\
\hline $\mathrm{R} 1$ & 21.80 & 1.67 & $\mathrm{R} 1$ & 22.16 & 1.87 \\
\hline \multicolumn{3}{|c|}{ UGC 7298} & \multicolumn{3}{|c|}{ UGC 8320} \\
\hline B1 & 21.93 & 0.20 & B1 & 19.37 & 0.17 \\
\hline $\mathrm{B} 2$ & 22.45 & 0.11 & $\mathrm{~B} 2 *)$ & 20.21 & $(0.21)$ \\
\hline B3 & 22.47 & 0.07 & B3 & 20.76 & 0.08 \\
\hline \multirow[t]{3}{*}{$\mathrm{R} 1$} & 23.33 & 1.60 & B4 & 21.10 & 0.00 \\
\hline & & & B5 & 21.40 & 0.12 \\
\hline & & & $\mathrm{R} 1$ & 22.97 & 2.30 \\
\hline
\end{tabular}

$(6,7)$ - the mean magnitude and mean colour of the three brightest blue stars;

$(8,9)$ - the distance modulus of the galaxy via blue stars and red one;

(10) - the galaxy distance (in Mpc), corresponding to the blue modulus. Below we note some properties of the galaxies observed.

NGC 4163 = UGC 7199. This blue compact galaxy has a quite regular shape on its faint isophotes. The integrated colour index increases smoothly from 0.41 in the center up to 0.53 within the largest visible radii, which may indicate the presence of two stellar populations in the galaxy. The brightest blue object $(=\mathrm{B} 0)$ has a starlike profile. However, we excluded it as a probable multiple system when the distance was derived.
NGC $4190=$ UGC $7232=$ VV 104. The galaxy seems to contain two overlapping regions of star formation. For this reason Vorontsov-Velyaminov (1959) included this peculiar object into the Atlas of interacting galaxies. The brightest blue stars are concentrated in the SW part of the galaxy. The distance estimate via the brightest red star (R1) agrees well with that from the blue ones.

UGC 7298. This small blue galaxy of a low surface brightness is distinctly resolved into stars, despite the fact that all of them are fainter than $V=21.5 \mathrm{mag}$. The galaxy distance, which is determined both via three blue stars and via the brightest red one, exceeds distances of both previous galaxies by more than two times. The distance estimate, 8.6 Mpc, is unusual for a galaxy with a radial velocity of only $+253 \mathrm{~km} / \mathrm{s}$.

UGC $7577=$ DDO 125. This and the next two galaxies are in the list of dwarf irregular galaxies of van den Bergh (1966). In spite of the large dimension of the galaxy $(4.2 \times 2.4$ arcmin $)$, almost all its brightest stars are concentrated within our CCD frame. From the three brightest blue stars the galaxy distance modulus is 28.42 mag. The brightest red star (R1) yields $28.71 \mathrm{mag}$, which is in agreement with the blue modulus. However, its colour index, 1.58 , does not formally satisfy the condition $B-V>1.6$ adopted above.

UGC $8308=$ DDO 167. The galaxy has an asymmetric shape and a low surface brightness. Its integral colour index increases smoothly from 0.29 in the central region up to 0.48 within the faintest isophotes. The distance estimates from the blue stars and the red one agree well with each other. But we have to note a large scatter in the apparent magnitudes of red stars, which makes the red modulus rather unreliable.

UGC $8320=$ DDO 168. From the six considered galaxies this is the only one that have already been resolved into stars. According to Bresolin et al. (1993) its distance is $3.3 \mathrm{Mpc}$. Photometry of 86 brightest stars in $B, R$ bands throughout the galaxy body was carried out by Hopp \& Schulte-Ladbeck (1995). For the common stars in the $B$ band we find good agreement between our and their photometry. Because of its large dimension, 
$3.7 \times 1.4$ arcmin, a large fraction of the galaxy stars fall outside our CCD frame. Therefore, estimating its distance via blue stars, we took the star No. 44 from Hopp \& Schulte-Ladbeck's list as the second brightest blue star, B2. The derived distance value, 4.0 Mpc, exceeds slightly the estimate of Bresolin et al. (1993).

\section{Summary}

For six irregular dwarf galaxies having radial velocities $V_{0}<300 \mathrm{~km} / \mathrm{s}$ and situated in the nearby CVn cloud we derived large-scale CCD images in the $B, V$ bands. All the galaxies are clearly resolved into stars. Five of them have been resolved for the first time. Using the luminosity of the brightest blue (and, sometimes, red) stars as a distance indicator, we determined the distances to these galaxies. Except for UGC 7298 with $D=8.6 \mathrm{Mpc}$, the distances lie in the range of $[3.5-4.8] \mathrm{Mpc}$. If the majority of the CVn cloud members take part in the general cosmologic expansion, the objects with the lowest radial velocities are expected to be located on the near side of the cloud. The data derived here do not contradict such a suggestion.

With the distances we have measured all the six galaxies are actually dwarf systems, whose absolute magnitudes lie between -12.3 and -15.6 mag. Judging by their total colours, $(B-V)_{\mathrm{t}}=[0.31-0.48]$, all the galaxies are at a stage of active star formation. Two of the six, NGC 4163 and UGC 8308, show a colour increase from the centre toward the periphery, which may indicate the presence of an old stellar population. Accumulation of data on the structure and population of dwarf galaxies in the nearest $\mathrm{CVn}$ cloud, as well as on their location with respect to other members of the complex, will allow to understand the conditions which affect the evolutional status of dwarf stellar systems.

Acknowledgements. This work was supported by INTASRFBR grant No. 95-IN-RU-1390 and RFBR grant No. 97-0217163 .

\section{References}

Bresolin F., Capaccioli M., Piotto G., 1993, AJ 105, 1779 de Vaucouleurs G., 1978, ApJ 224, 14

Georgiev Ts.B., 1995, WGWFI Newslett. 54, 611

Georgiev Ts.B., Karachentsev I.D., Tikhonov N.A., 1997, Lett. AZh, 23 (accepted)

Hopp U., Schulte-Ladbeck R.E., 1995, A\&ApS 111, 527

Karachentsev I.D., Makarov D.I., 1996, AJ 111, 794

Karachentsev I.D., Tikhonov N.A., 1994, A\&A 286, 718

Landolt A.U., 1992, AJ 104, 340

Makarova L.N., Karachentsev I.D., Georgiev Ts.B., 1997, Lett. AZh 23, 435

Makarova L.N., Karachentsev I.D., Takalo L., Heinamaki P., Valtonen M., 1998, A\&ApS (accepted)

Paturel G., Bottinelli L., Fouque P., Gouguenheim L., 1992, Catalogue of Principal Galaxies: PGC-ROM, Observatoire de Lyon

Sandage A.R., Tammann G.A., 1974, ApJ 190, 525

Treffers R.R., Richmond M.W., 1989, PASP 101, 725

Tully R.B., Fisher J.R., 1977, A\&A 54, 611

van den Bergh S., 1966, AJ 71, 922

Vorontsov-Velyaminov B.A., 1959, Atlas and Catalogue of interacting galaxies, Moscow State Univ., Moscow 